\title{
Spontaneous triple-vessel coronary artery dissection in a patient with Ehlers-Danlos Type IV
}

\author{
Andres E. Carmona-Rubio*, Narasa Madam, Jennifer Lang, Susan Graham and Umesh Sharma \\ Department of Medicine, University at Buffalo, State University of New York, School of Medicine, Buffalo, NY
}

\begin{abstract}
Background: Ehlers-Danlos syndrome type IV -vascular type- is an inherited connective tissue disorder characterized by several clinical features: easy bruisability, translucent skin, acrogeria and uterine, intestinal or arterial rupture. The arteries most frequently involved are the iliac, splenic, renal arteries and the aorta. Diagnosis is made utilizing clinical criteria (Beighton) and collagen gene mutation testing via skin biopsy.

Case: We present a case of a 53 year old, Caucasian female with Ehlers-Danlos Type IV and history of myocardial infarction secondary to aortic dissection and coronary artery dissection (left anterior descending, left circumflex and right coronary artery) with subsequent coronary artery bypass grafting. The patient arrived to the emergency room with left-sided chest pain radiating to her left arm. Electrocardiography showed ST-segment depressions in leads I and aVL with T-wave inversions in leads V4-V6. On laboratory she had troponin elevation peaking at 3.35. Coronary computerized tomographic angiography was performed due to concerns of coronary artery rupture with invasive coronary angiography; revealing a slit-like narrowing of approximately $50 \%$ of her proximal left anterior descending artery and multiple ectatic to aneurysmal coronary artery segments involving the three major coronary vessels. Our approach was to pursue conservative management, optimizing her therapeutic regimen to prevent chest pain recurrence.
\end{abstract}

Conclusion: Spontaneous coronary artery dissection is extremely rare and when it occurs case-reports have shown that it affects the left anterior descending artery. In this case we present a patient with triple vessel involvement which to the best of our knowledge has only been reported once. We believe that patients with EhlersDanlos Type IV who present with chest pain, should be evaluated with non-invasive imaging (i.e. CCTA or Cardiac MRI), as invasive procedures could potentially be more harmful than beneficial.

\section{Background}

Ehlers-Danlos syndrome (EDS), type IV is usually associated with arterial ruptures. Vascular rupture or dissection and gastrointestinal perforation or organ rupture are the presenting signs in $70 \%$ of adults. The sites of arterial rupture most frequently affected are the thorax and abdomen (50\%), head and neck (25\%), and extremities (25\%) [1]. The vessels that have been reported to be involved are: iliac, splenic, mesenteric, carotid, renal arteries and the aorta. There are few reported cases with coronary artery dissection in EDS Type IV, but it is even rarer when there is involvement of the three major coronary vessels [2-4].

\section{Case report}

This is a case of a 53 year old, Caucasian female, whose history of present illness started 15 years/ago when she went to an outside facility complaining of left-sided chest pain. Electrocardiography at that time showed an acute anterior ST-segment elevation myocardial infarction (STEMI) and was treated with thrombolytics and transferred for urgent intervention to a STEMI referral center. Invasive coronary artery angiography (ICA) revealed left anterior descending artery (LAD) dissection. She was taken emergently for coronary arterial bypass grafting (CABG), where an attempted left internal mammary artery (LIMA) to LAD grafting was performed. On her post-operative day \#4 she had recurrent chest pain and was re-studied by ICA, demonstrating that the LIMA was closed and the right coronary artery (RCA) and left circumflex artery (LCx) had spontaneously dissected after contrast dye was injected during the procedure; having now triple vessel dissection. There was no coronary stenting and she was treated with conservative medical management (Beta blocker, dual antiplatelet therapy -aspirin and clopidogrel-, angiotensin receptor blocker and statins) and discharged to home.

Spontaneous coronary artery dissection (SCAD) is a rare condition which raised suspicion of an underline condition which could have served as a predisposing factor. Although there were no obvious clinical signs on physical examination to suspect EDS-IV, a biopsy of her skin was obtained for genetic testing. The results revealed the presence of an alteration in the structure of the proal(III) chains of type III procollagen encoded by the COL3A1 allele, which has been associated with Ehlres-Danlos Type IV, the vascular type.

Eight years later of her EDS diagnosis the patient had left internal carotid dissection treated conservatively and 2 years after she had a repeat myocardial infarction (MI). Coronary computerized tomographic angiography (CCTA) at that time showed patent LIMA to $\mathrm{LAD}$, prior $\mathrm{LAD}$ dissection, focal residual dilatation of the proximal LAD, occluded mid-LAD beyond LIMA graft, dissection changes in the LCx and occlusion of the RCA distal to the crux. Cardiac magnetic

Correspondence to: Dr. Andres E Carmona-Rubio, MD, Department of Medicine, Erie County Medical, Center, David K. Miller Building, 462 Grider St., Buffalo, NY 14215, USA, Tel: (716) 898-4806; Fax: (716) 898-3279; E-mail: carmonarubio@gmail.com

Key words: Ehlers Danlos Type IV, Ehlers Danlos vascular type, coronary cardiac tomographic angiography, coronary artery dissection

Received: October 10, 2015; Accepted: November 12, 2015; Published: November 20, 2015 
resonance imaging (MRI) showed a scar of the apex with focal akinesis and global hypokinesis with a left ventricular ejection fraction (LVEF) of $30 \%$. She was again treated conservatively and a follow-up multi gated acquisition (MUGA) scan 5 months later showed an EF of $49 \%$ without any focal wall motion abnormalities.

The patient presented to our institution complaining of left-sided pressure like chest pain radiating to the left arm and back relieved by the administration of hydromorphone. Physical exam was only remarkable for skin paleness. Laboratory results revealed an elevated troponin I, peaking at $3.35 \mathrm{ng} / \mathrm{ml}$. Twelve-lead electrocardiography was noted to have ST segment depressions in leads I and aVL with T-wave inversions in leads V4, V5 and V6 (Figures 1A-1B); meeting criteria for non-ST elevation MI (NSTEMI). Two dimensional transthoracic echocardiography (TTE) showed mild to moderately reduced left ventricular (LV) systolic function with an LVEF in the range of 35$45 \%$ and global hypokinesis in the LV.

Non-invasive imaging with CCTA was performed showing a slitlike narrowing of approximately $50 \%$ of the proximal LAD at its origin with complete occlusion of proximal to mid-LAD just distal to the first septal perforator branch. Multiple ectatic to aneurysmal coronary artery segments involving all 3 main coronary arteries (LAD, LCx and RCA) and the absence of atherosclerotic coronary artery disease. (Figure 2A-C) Computerized tomographic angiography of the chest and abdomen was done and revealed no evidence of aortic dissection or elsewhere. Scattered pseudoaneurysms involving the right vertebral artery, splenic artery, common hepatic artery, right renal artery and right sided short gastric artery.

A multidisciplinary discussion about the possibility of any further invasive diagnostic workup was done and the consensus was that the risks outweighed the benefits, as her risks for further dissection and mortality exceeded $50 \%$ by ICA. Conservative management with optimal medical treatment to prevent chest pain recurrence with ranolazine and close follow-up in the clinic.

\section{Discussion}

Spontaneous coronary artery dissection was first described in 1931. Since then 480 cases have been reported on MEDLINE. It typically affects young women and otherwise healthy individuals. The incidence of SCAD is approximately $1-4 \%$ in patients who present with acute coronary syndrome. Average age for the presentation of SCAD is 42 years, though there have been cases reported from the first decade to the seventh decade of life. Approximately $80 \%$ of SCAD patients are female and, of those $20 \%$ to $25 \%$ of cases occur in the peri-partum period [5-7].

The pathogenesis of SCAD is not yet fully comprehended. The condition occurs when blood collects within the media of the coronary artery or between the media and external elastic lamina, causing compression of the true lumen of the artery. The initial event leading to dissection is not clear, and no single factor has been found to be causative. Many of the proposed mechanisms pertain to changes in vascular wall properties that lead to weakening of the media and connective tissue. These include changes in smooth-muscle-cell metabolism, the effect of proteases released from eosinophilic infiltrates, and pregnancy-related changes in connective tissue [8].

Conditions which are associated with SCAD are: pregnancy or postpartum period (most prevalent), fibromuscular dysplasia, hypertensive crisis, coronary vasospasm, systemic inflammatory diseases (poliarteritisnodosa, systemic lupus erythematous and Churg-Strauss syndrome), neurofibromatosis, connective tissue abnormalities/monogenetic mutations (pseudoxantomaelasticum, autosomal dominant polycystic kidney disease, Loeys-Dietz syndrome, Marfan syndrome and type-IV EDS) and pharmacologic agents (cocaine and cabergoline) [7-9].

The Ehlers-Danlos syndromes are a heterogeneous group of heritable connective tissue disorders characterized by articular hypermobility, skin extensibility, and tissue fragility. Seven types of EDS have been described and their estimated prevalence varies between $1 / 10,000$ and $1 / 25,000$, without any ethnic predisposition. EhlersDanlos type IV is considered to be the vascular or arterial-ecchymotic type and it accounts for approximately $5-10 \%$ of the cases $[10,11]$. This disease is diagnosed by performing genetic testing and skin biopsies to analyze collagen obtained from cultured fibroblasts [12].

Amongst arterial dissections in vascular EDS they tend to occur in medium-large caliber arteries. The most frequently associated arteries are: iliac (26\%), aorta (11\%), renal (8\%), carotid (7\%) and celiac artery (7\%). Coronary artery dissections are extremely rare. The vessel most frequently involved, according to the literature is the LAD. Although publications regarding the RCA and LCx are also available $[11,13]$.
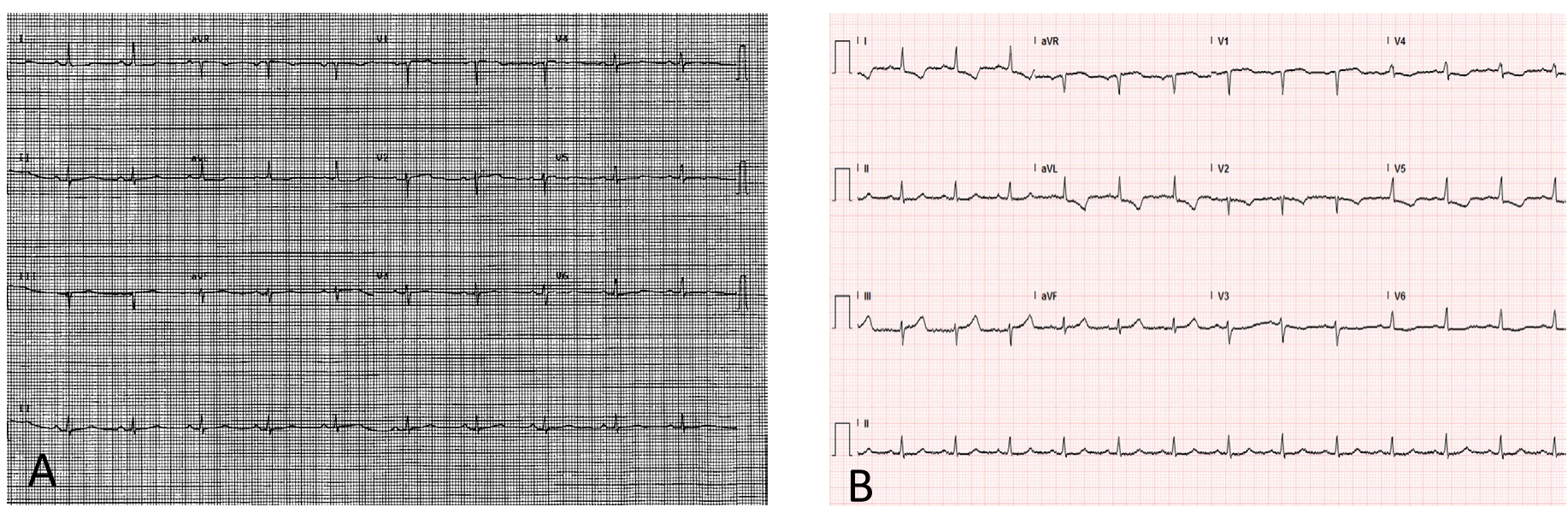

Figure 1. Panel A. Prior electrocardiogram. Panel B. Electrocardiogram on admission: ST segmentdepressions in leads I andaVL with T-wave inversions in leads V4, V5 and V6. 

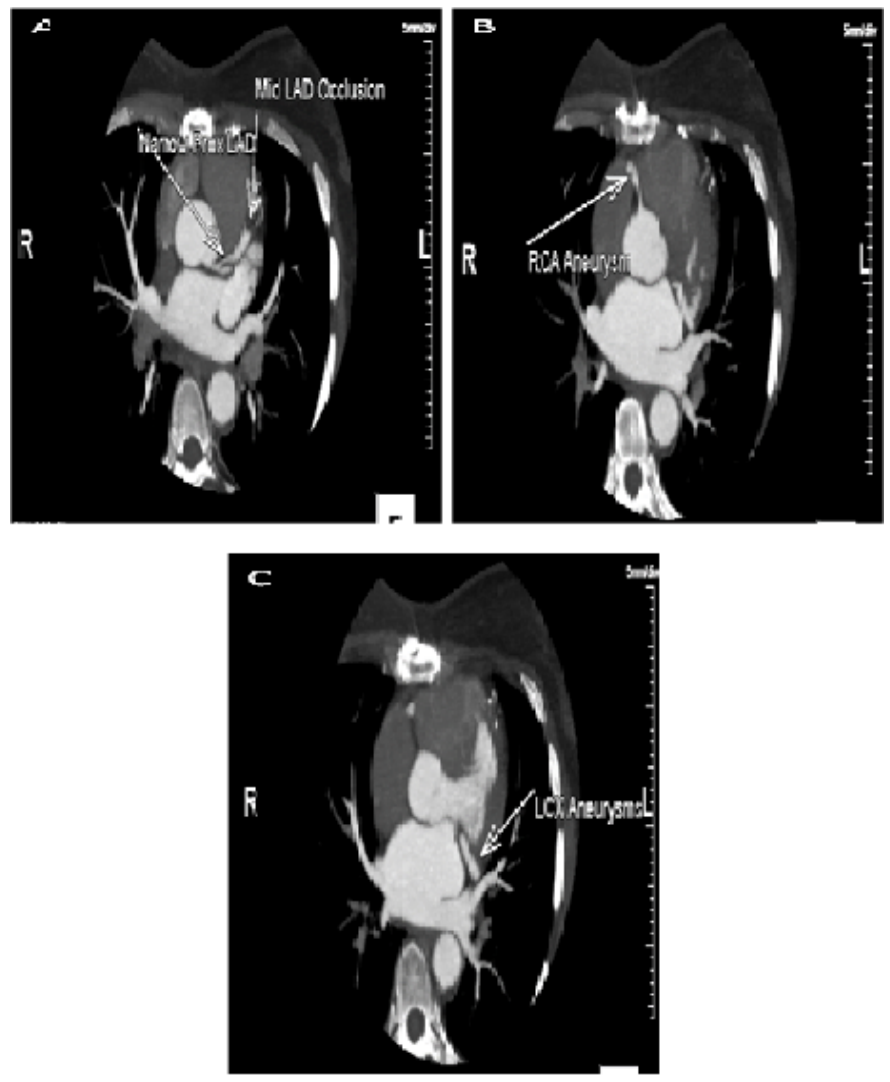

Figure 2. Panel A. Slit-like narrowing (approximately 50\%) of the proximal LAD at its origin with complete occlusion of the proximal to mid-LAD just distal to the first septal perforator branch.Minimal distal flow beyond the occlusion likely contributed by smal collaterals or the bypass graft to distal LAD. Panel B. RCA arises from the right coronary sinus of Valsalva. We can see a short aneurysmal segment of mid-RCA without significant luminal narrowing. Panel C. No atherosclerosis or luminal narrowing. Mid left circumflex has a short ectatic segment without evidence of luminal narrowing or dissection.

Guidelines to treat SCAD have not been established. In the event that they present with an acute MI with ongoing ischemia, the first objective should be to restore normal coronary flow. Fibrin-specific thrombolytic therapy is discouraged because it may result in further propagation of the dissection. Primary percutaneous coronary intervention (PCI) remains the reperfusion strategy of choice. If the vessel is open and the flow normalized at the time of angiography, it is defendable to treat the dissection conservatively. Good angiographic and clinical outcomes have been described with medical treatment only. With conservative measures, coronary artery dissections have even shown complete angiographic resolution after a year [12].

Coronary artery revascularization in vascular EDS either using PCI or CABG is challenging due to the arteries friability. A small number of cases have been reported describing the friability of the vessels and associated complications. However, when it is a large epicardial vessel, placement of a stent will often be the treatment of choice. In case of multi-vessel or left main involvement, several case reports also describe treatment with urgent surgical revascularization $[12,14]$.

\section{Conclusion}

Spontaneous coronary artery dissections are rare, multifactorial and complex phenomenon's. The presentation can vary from chest pain to sudden cardiac death. This pathology is even more infrequent if it is associated with EDS-IV. Angiography is the diagnostic and therapeutic method regularly utilized in these patients. Non-invasive imaging, CCTA, can be indicated, as it helps with the detection and follow-up of coronary artery involvement or/and progression of known dissections. It is also, a safer modality for patients with EDS-IV, as it would have less risk of complications. Conservative management should be the therapy of choice if possible in all these patients. There is promising evidence with celiprolol (cardioselective, $\beta 2$ agonist with vasodilator properties) as a preventative option [15]. Nevertheless, the optimum treatment remains unclear to avoid or treat vascular complications.

\section{References}

1. Pepin MG BP (1999) Ehlers-Danlos Syndrome Type IV. Internet ed. Seattle (WA): University of Washington, Seattle.

2. Adès LC, Waltham RD, Chiodo AA, Bateman JF (1995) Myocardial infarction resulting from coronary artery dissection in an adolescent with Ehlers-Danlos syndrome type IV due to a type III collagen mutation. Br Heart $J$ 74: 112-116. [Crossref]

3. Athanassiou AM, Turrentine MA (1996) Myocardial infarction and coronary artery dissection during pregnancy associated with type IV Ehlers-Danlos syndrome. Am J Perinatol 13: 181-183. [Crossref]

4. HampoleCV,Philip F, Shafii A, Pettersson G, Anesi GL, et al. (2011) Spontaneous coronary artery dissection in Ehlers-Danlos syndrome. Ann ThoracSurg 92: 18831884. [Crossref]

5. Hayes SN (2014) Spontaneous coronary artery dissection (SCAD): new insights into this not-so-rare condition. Tex Heart Inst J 41: 295-298. [Crossref]

6. Shamloo BK, Chintala RS, Nasur A, Ghazvini M, Shariat P, et al. (2010) Spontaneous coronary artery dissection: aggressive vs. conservative therapy. J Invasive Cardiol 22: 222-228. [Crossref]

7. HC P (1931) Dissecting aneurysm of a coronary artery in a woman aged 42. BMJ

8. Klein J,Hakimian J, Makaryus AN (2012) Spontaneous right coronary artery dissection: causing myocardial infarction in a 36-year-old woman. Tex Heart Inst $J$ 39: 95-98. [Crossref]

9. Saw J RD, Starovoytov A, Fox R, Buller CE (2013) Spontaneous coronary artery dissection: prevalence of predisposing conditions including fibromuscular dysplasia in a tertiary center cohort. JACC CardiovascInterv 6:44-45.

10. Beighton P,De Paepe A, Steinmann B, Tsipouras P, Wenstrup RJ (1998) Ehlers-Danlos syndromes: revised nosology, Villefranche, 1997. Ehlers-Danlos National Foundation (USA) and Ehlers-Danlos Support Group (UK). Am J Med Genet 77: 31-37. [Crossref]

11. Nakamura M, Yajima J, Oikawa Y, Ogasawara K, Uejima T, et al. (2009) Vascular Ehlers-Danlos syndrome--all three coronary artery spontaneous dissections. $J$ Cardiol 53: 458-462. [Crossref]

12. Tanis W,Stella PR, Kirkels JH, Pijlman AH, Peters RH, et al. (2008) Spontaneous coronary artery dissection: current insights and therapy. Neth Heart $J$ 16: 344-349. [Crossref]

13. Zilocchi M, Macedo TA, Oderich GS, Vrtiska TJ, Biondetti PR, et al. (2007) Vascular Ehlers-Danlos syndrome: imaging findings. AJR Am J Roentgenol 189: 712-719. [Crossref]

14. Zago AC, Matte BS (2013) Friable but treatable: coronary artery dissections in EhlersDanlos syndrome. Catheter CardiovascInterv 81: 75-79. [Crossref]

15. Ong KT PJ, De Backer J, Bozec E, Collignon P, Emmerich J, et al. (2010) Effect of celiprolol on prevention of cardiovascular events in vascular Ehlers-Danlos syndrome: a prospective randomised, open, blinded-endpoints trial. Lancet 376:1476-1484.

Copyright: (C2015 Ganguly S. This is an open-access article distributed under the terms of the Creative Commons Attribution License, which permits unrestricted use, distribution, and reproduction in any medium, provided the original author and source are credited. 